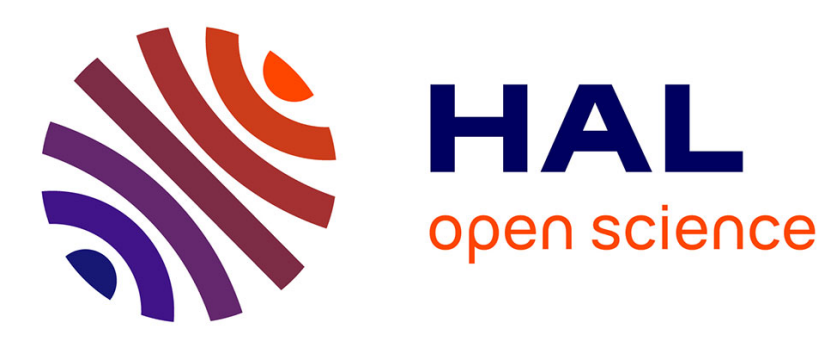

\title{
Le système d'activité: retour sur un concept pour étudier l'agriculture en famille
}

Pierre Gasselin, Michel Vaillant, Benjamin Bathfield

\section{To cite this version:}

Pierre Gasselin, Michel Vaillant, Benjamin Bathfield. Le système d'activité: retour sur un concept pour étudier l'agriculture en famille. L'agriculture en famille: travailler, réinventer, transmettre, EDP Sciences, 382 p., 2014, 978-2-7598-1192-2 978-2-7598-1765-8. 10.1051/978-2-7598-1192-2.c007 . hal-02796832

\section{HAL Id: hal-02796832 \\ https://hal.inrae.fr/hal-02796832}

Submitted on 5 Jun 2020

HAL is a multi-disciplinary open access archive for the deposit and dissemination of scientific research documents, whether they are published or not. The documents may come from teaching and research institutions in France or abroad, or from public or private research centers.
L'archive ouverte pluridisciplinaire HAL, est destinée au dépôt et à la diffusion de documents scientifiques de niveau recherche, publiés ou non, émanant des établissements d'enseignement et de recherche français ou étrangers, des laboratoires publics ou privés. 


\title{
Le système d'activité. Retour sur un concept pour étudier l'agriculture en famille
}

\author{
Pierre Gasselin', Michel Vaillant ${ }^{2}$, Benjamin Bathfield ${ }^{3}$
}

\section{Introduction ${ }^{4}$}

Il y a tout juste vingt ans, Paul et al. (1994) publiaient dans les Cahiers de la Recherche Développement un article où ils présentaient le chemin qui les avait conduits à élaborer un concept pour rendre compte de la réalité complexe des agricultures caribéennes : le système d'activité ${ }^{5}$. Celui-ci devait servir à mieux saisir la rationalité de familles agricoles dont la principale caractéristique résidait dans le fait de combiner au cours de l'année plusieurs activités, agricoles et non agricoles. Si Cellier et Marquié (1980) l'avaient convoqué avant eux, depuis, le concept appliqué à des problématiques agricoles et rurales a eu un usage limité, restant surtout le fait d'une communauté scientifique française (Dedieu et al., 1999; Chia et al., 2006 ; Gaillard et Sourisseau, 2009 ; Fiorelli, 2010 ; Gasselin, 2012b ; Gasselin et al., 2012 ; Bathfield, 2013 ; Bathfield et al., 2013 ; Mundler, 2013 ; Terrier et al., 2013).

1. INRA, UMR 951 Innovation, F-34060 Montpellier, France, pierre.gasselin@supagro.inra.fr

2. AgroParisTech, Chaire d'agriculture comparée, F-75005 Paris, France, mitchvaillant@yahoo.fr

3. ECOSUR, Unidad de San Cristóbal de las Casas, Chiapas, México, benjaminbathfield@yahoo.com.mx

4. Ce travail a bénéficié d'une aide de l'Agence nationale de la recherche dans le cadre du programme Systerra, portant la référence ANR-09-STRA-04. Il remobilise et complète une communication présentée en 2012 à la conférence de l'International Farming System Association (Gasselin et al., 2012).

5. Le mot " activité " est, lorsqu'il est associé à celui de système, délibérément et systématiquement employé au singulier, à l'instar de celui de "production " dans système de production agricole. Ce dernier désigne la manière complexe de produire des biens, animaux et végétaux, diversifiés : le mot production est ainsi pris dans son sens générique (la production agricole). Il en est de même pour le système d'activité, qui renvoie à la manière de combiner différentes activités, d'où l'emploi du mot " activité " au singulier (Vaillant, 2013).

Ce chapitre de l'ouvrage L'agriculture en famille : travailler, réinventer, transmettre est publié en Open Access sous licence creative commons CC-BY-NC-ND permettant l'utilisation non commerciale, la distribution, la reproduction du texte, sur n'importe quel support, à condition de citer la source. 
À peu près simultanément, l'approche par les Rural Livelihoods, devenue rapidement celle des Sustainable Rural Livelihoods (SRL), s'impose de façon quasi hégémonique sur la recherche anglophone pour et sur le développement des populations rurales pauvres, et ce depuis les travaux fondateurs de Chambers et Conway (1991), Scoones (1998), Ellis (1998, 2000), Farrington (1999) et Bebbington (1999) dans les années 1990. Ce sont plus de vingt ans de travaux qui donnent lieu à une production pléthorique : fin 2013, le Web of Science indexe plus de 2500 références dont le titre ou les mots-clés portent sur les Rural Livelihoods. Ces dernières années, plusieurs auteurs (O'Laughlin, 2004 ; Gaillard et Sourisseau, 2009 ; Gasselin, 2012a), dont un de ses pères fondateurs, Ian Scoones (2009), font toutefois une analyse critique de la trajectoire du concept et pointent plusieurs lacunes et ambiguïtés, notamment sur la façon dont les SRL traitent des questions de connaissances et de savoirs, de politiques, de changement d'échelle ou encore d'approche diachronique.

Dans le monde entier, de nombreux auteurs constatent l'importance des combinaisons d'activités dans et autour de l'activité agricole et de l'exploitation agricole (Laurent et al., 1998 ; Ellis, 2000 ; de Janvry et al., 2005 ; Malézieux et Moustier, 2005 ; Dufumier, 2006 ; Haggblade et al., 2007). De cette observation d'une diversification des productions et des activités découle des enjeux de connaissance mais aussi des défis politiques et opérationnels. Conçus pour s’appliquer à ces problématiques, les concepts de système d'activité et de Sustainable Rural Livelihoods sont à la fois proches, par leurs objets - souvent l'exploitation agricole tenue par une famille pluriactive - et les problématiques, et distantes par les communautés relativement cloisonnées qui les mobilisent et par les fondamentaux théoriques qui les soustendent. L'année internationale de l'agriculture familiale constitue un moment opportun pour revenir sur le concept de système d'activité avancé voici deux décennies, en tant qu'outil analytique en vue d'étudier et d'accompagner les pratiques et les décisions d'acteurs (individu, foyer, ménage, famille, petite entreprise, exploitation agricole, etc.) qui mettent en œuvre au moins une activité agricole (production végétale et/ou animale). Nous en proposons ici une version revisitée, au regard des travaux existants sur le sujet, mais aussi à partir de nos travaux de terrains conduits en France et en Amérique latine.

Les recompositions de l'exploitation agricole sont largement analysées dans cet ouvrage, notamment par des processus qui concourent à la dilution du caractère familial. Ces mutations invitent à décaler le regard pour resituer l'exploitation agricole comme un maillon d'une combinaison d'activités, de ressources et d'acteurs, représentée par le système d'activité, échelle à laquelle s'établit et s'interprète «le domaine de cohérence de la rationalité de l'agriculteur " (Paul et al., 1994). Nous souhaitons montrer que le concept de système d'activité garde aujourd'hui toute sa pertinence, en tant qu'outil d'analyse, pour se saisir de l'évolution des agricultures familiales, afin de comprendre les décisions prises en leur sein, en interroger le sens, et pour produire de la connaissance utile à des décideurs politiques, des acteurs du développement et des organisations paysannes. Après un positionnement théorique et empirique, nous présentons le concept de système d'activité, les conditions de 
son emploi et concluons sur les proximités et divergences avec les Sustainable Rural Livelihoods.

\section{Des ancrages théoriques et empiriques}

Les fondements théoriques du système d'activité intègrent de nombreux champs de recherche, notamment dans le domaine agricole et rural, sur l'approche systémique, les ressources et les processus d'action et de décision individuelle, sur l'action collective, l'activité et le travail, sur l'innovation et le développement. Cette première partie s'attache à reconnaître les différents socles théoriques sur lesquels repose le concept de système d'activité. Ce panorama s'est construit au fur et à mesure, au gré des problématiques abordées en mobilisant ce concept dans différents contextes et pour répondre à différentes questions. Il n'y a donc pas nécessairement de filiation intellectuelle entre les auteurs cités en référence. Pour autant, nos postulats théoriques s'inscrivent dans un champ de cohérence. Si les diverses théories mobilisées ne peuvent se prévaloir de "faire système ", elles n'en sont pas pour autant éclectiques. La seconde partie de cette section présente les principales problématiques des cinq opérations de recherche dans lesquelles le concept de système d'activité a été mobilisé.

\section{Principales filiations théoriques}

Nous nous inscrivons dans l'héritage d'une pensée systémique (Crozier et Friedberg, 1977 ; Morin, 1990) appliquée aux problématique agricoles et rurales (Brossier et al., 1990 ; Sebillotte, 1996 ; Cochet, 2011 ; Norman, 2002). Plusieurs auteurs appréhendent la combinaison d'activités dans une perspective systémique (Aubry, 2007 ; Chayanov, 1990 ; Curie et al., 1990 ; Gaillard et Sourisseau, 2009 ; Mundler, 2013) en reconnaissant que "ce méta-système que nous nommons système d'activité constitue le véritable domaine de cohérence des pratiques et des choix des agriculteurs" (Paul et al., 1994). Reconnaître le caractère systémique de la combinaison d'activités conduit à expliciter ses propriétés émergentes (la combinaison d'activités est plus que la simple somme des activités) et à examiner avec attention les interactions (entre les activités, mais aussi entre les ressources mobilisées et entre le système et son environnement).

Nous considérons que l'acteur du système d'activité est doté d'une polyrationalité, d'une subjectivité et d'une intentionnalité selon des théories de l'action et de la décision qui résolvent les oppositions sociologiques de type "structurelagency ". L'acteur du système d'activité (ses décisions et ses pratiques) n'est ni entièrement déterminé par des structures et des institutions le dépassant (holisme et structuralisme de Durkheim), ni pleinement et librement créateur rationnel de ses activités (individualisme méthodologique hérité de Weber). De nombreux auteurs des sciences sociales et techniques inspirent nos travaux sur la décision et les pratiques 
tels Bourdieu (1980) et Boltanski et Chiapello (1999), certains plus spécialisés dans le champ de l'agriculture comme Osty (1978), Brossier et al. (1990), Darré (1999) et Fiorelli (2010).

Le système d'activité est une représentation abstraite, schématique et simplifiée de la combinaison d'activités effectivement mise en œuvre par un acteur. L'étudier impose dès lors de définir ce que l'on désigne par activité/s. Plusieurs théories étayent nos conceptions de l'activité humaine. Nous nous reconnaissons notamment dans les théories de Arendt (1994) qui en distingue trois dimensions fondamentales : 1) la dimension du "Travail » qui correspond à la vie économique et domestique (la satisfaction des besoins), 2) la dimension de "l'Euvre " autrement dit de la création personnelle (fabrication d'un monde commun et d'objets d'usage plus que de consommation), et 3) la dimension de "l'Action " qui est celle de la vie politique et associative dans l'espace public et social. Cette conception de l'activité élargit la proposition méthodologique de description des systèmes d'activité de Curie et al. (1990) qui distinguent trois " domaines de vie " (introduits par Lanneau et al., 1987) : vie familiale et domestique, vie professionnelle, vie personnelle et sociale ${ }^{6}$. En effet, Arendt resitue l'individu et son activité dans un univers de sens et un espace politique permettant d'interroger la « condition de l'homme moderne » dans son travail, dans son œuvre créatrice et au regard de sa liberté.

De nombreuses recherches sur le travail, que l'on ne confond pas avec l'activité ou la tâche, ont aussi été mobilisées, notamment les travaux inspirés de Dejours qui précise la dimension subjective du travail en reconnaissant que « travailler c'est mobiliser son corps, son intelligence, sa personne, pour une production ayant valeur d'usage » (Dejours, 1998). L'activité agricole est bien entendu reconnue dans sa multifonctionnalité (Caron et al., 2008). Elle ne crée donc pas que des valeurs marchandes.

Décider et conduire une activité suppose des ressources. Quelques auteurs majeurs orientent notre pensée sur les ressources du système d'activité, notamment Boserup (1970) et son explication de l'évolution historique des techniques agricoles, Polanyi (1983) et son approche sociale de l'échange qui n'est pas seulement le fait du marché ou de l'État, Mazoyer \& Roudard (1997) ainsi que Cochet et al. (2007) pour leur théorie des systèmes agraires, Gumuchian et Pecqueur (2007) et leur approche des

6. Cette catégorisation de l'activité en " domaines de vie " pose des difficultés méthodologiques pour en délimiter les périmètres. À titre d'exemple, la "vie sociale " ne se limite pas aux "domaines" personnels et familiaux, elle est aussi fondamentale dans l'activité " professionnelle ". Par ailleurs, les catégories "vie domestique » et "vie professionnelle " sont polysémiques et ne sont pas étanches. Rappelons que les notions de "profession » ou de "professionnel » renvoient à des critères très divers selon les mondes sociaux (en tant que catégorie d'action et identitaire) et selon les disciplines (en tant que catégorie analytique, par exemple dans la sociologie des professions). Ces critères sont notamment les statuts juridiques et sociaux, le temps passé, le fait que l'activité génère ou non une rémunération, la reconnaissance d'une compétence, un degré de spécialisation, l'appartenance à une corporation de métiers ou à un syndicat, la possession d'un diplôme, etc. 
ressources territoriales, Sen (2008) avec le concept de capacité et de ses contributions à la théorie du choix personnel et Ostrom et Baechler (2010) qui nous ont donné les critères pour penser l'action collective dans la gestion des ressources communes. Le système d'actvité s'inscrit dans une pensée socioéconomique et anthropologique qui reconnaît que l'acteur dispose de ressources marchandes et non-marchandes, certaines latentes (non-immédiatement disponibles ou pas mobilisées dans l'activité), d'autres activées (directement disponibles pour l'action et la décision). Certaines ressources sont matérielles et d'autres idéelles (les savoirs, l'autorité, etc.).

\section{Travaux empiriques}

Nous avons mobilisé le même concept de système d'activité sur des terrains aussi différents que la pampa argentine (Albaladejo et al., 2011 ; Albaladejo et al., 2012), la région des Salars des Andes boliviennes (Gasselin et al., 2010 ; Gasselin et Vaillant, 2010), les Andes australes de l'Équateur (Vaillant, 2008 ; Vaillant, 2013), l'Ouest du Guatemala (Bathfield, 2013 ; Bathfield et al., 2013) et la région LanguedocRoussillon en France (Gasselin, 2011 ; Gasselin, 2012b ; Terrier et al., 2013). Ces cinq opérations de recherche conduites depuis 2006 sont synthétisées dans le tableau 1. Elles ont fait l'objet de collaborations croisées et de travaux comparatifs dans le cadre de projets de recherche et de deux thèses de doctorat (Bathfield, 2013; Vaillant, 2013). Faute de place, il ne nous est pas possible d'illustrer chacun des éléments du cadre théorique à chaque fois par l'ensemble des cinq cas. Ne retenir qu'un seul cas en guise de fil d'Ariane des illustrations limiterait le spectre des utilisations possibles du concept. Aussi, nous avons préféré mobiliser les études de cas en sélectionnant celles qui apportent les meilleures illustrations quant aux diverses façons de mobiliser le concept. Nous invitons le lecteur désireux d'approfondir le sujet à consulter les articles et les thèses de doctorat cités en référence et qui rendent compte des cinq chantiers de recherche. 


\section{Le système d'activité : un concept}

\section{Définition}

Le système d'activité est un concept polysémique et transdisciplinaire, depuis la chimie à l'épidémiologie en passant par l'histoire ou l'économie. Nous le définissons comme un ensemble dynamique et structuré d'activités en interaction mises en œuvre par une entité sociale donnée en mobilisant des ressources disponibles dans un environnement écologique et social donné (Fig. 1). Ce faisant, nous n'attribuons pas au système d'activité et à l'entité sociale de finalités ou d'objectifs $a$ priori. Ceux-ci peuvent être déterminés par l'observateur qui interprète les décisions et les pratiques au regard d'une problématique et d'un référentiel disciplinaire et théorique (objectifs émergents) ou explicités par l'entité sociale (objectifs exprimés). L'application du concept de système d'activité à une réalité et à une problématique données impose donc de préciser le contenu des éléments constitutifs du système : entité sociale et activité/s considérée/s, registres de motivations de l'action et de la décision, types de représentations étudiées, ressources envisagées, composantes de l'environnement priorisées. Ces éléments doivent être définis en fonction de la question de recherche (qui précise une problématique, des disciplines et des cadres théoriques) et d'un contexte (historique, social, économique, technique, politique, culturel, etc.). Cet exercice de délimitation du champ de l'observation est une condition d'utilisation du concept et contribue à l'analyse. Quelle est l'entité sociale pertinente ? De quelle/s activité/s parle-t-on ? Comment pouvons-nous identifier et prendre en compte les différentes motivations (terme expliqué ci-dessous) des individus au sein de l'entité sociale? Quelles représentations doivent être prises en compte ? Quelles ressources doivent être examinées ? Comment délimiter l'environnement considéré ? Les "valeurs " du système d'activité du point de vue de l'entité sociale sont intrinsèquement liées aux motivations, représentations et aux types d'activités considérées. Les valeurs du système d'activité attendues par la communauté (la nation, les gouvernements locaux, les organisations de producteurs, etc.) sont souvent variées (Terrier et al., 2013). Par conséquent : quelles valeurs du système d'activité doivent être étudiées (le revenu, la qualité de vie, la production marchande, la production non marchande, le sens, la capacité d'adaptation, la vulnérabilité, la viabilité, les externalités environnementales et sociales, etc.) ? Nous précisons ci-dessous les enjeux et les raisonnements qui président au choix de ces unités d'observation. 


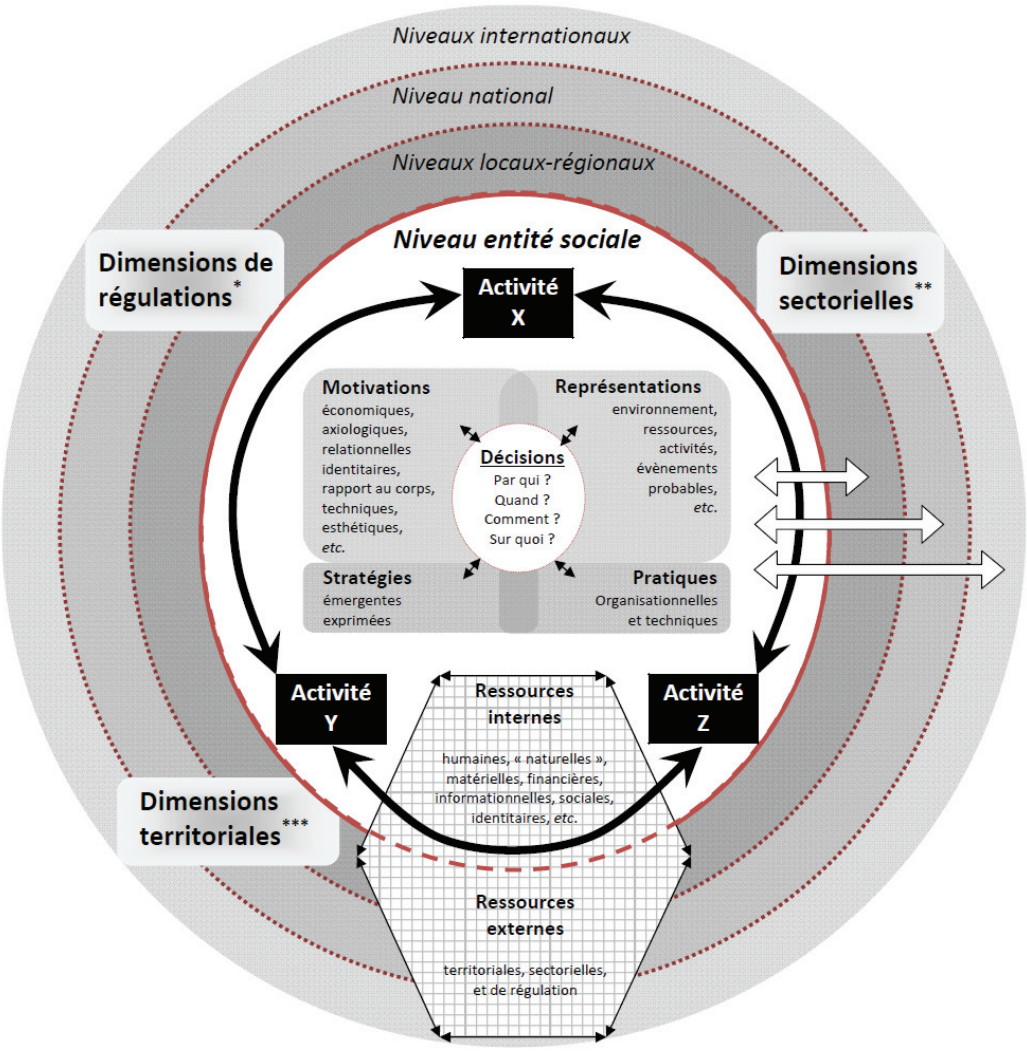

Dimensions de régulations*: Politiques, dispositifs de soutien et de normes publics et privés (règles, institutions, outils, etc.), gouvernance, rapports de force, etc.

Dimensions sectorielles**: Acteurs de la chaîne de valeur et des professions, marchés, régulations prix, acteurs et règles de la certification, etc. Identité (histoire, culture, patrimoine, limites, nom, etc.), organisation sociale et politique (coopératives, syndicats, collectivités locales, etc.), dimensions matérielles (conditions biophysiques et aménagement), organisation réticulaire (biens, personnes, services, argent, savoirs), projet d'une société sur son espace, etc.

Fig. 1. Le système d'activité (Source : les auteurs).

\section{Le périmètre contextualisé du système d'activité}

L'environnement en interaction avec le système d'activité est à la fois un contexte qui définit des opportunités, des atouts, des menaces et des contraintes, et un coproduit de l'entité sociale considérée et de ses activités. Nous y reconnaissons notamment des dimensions de régulation, des dimensions sectorielles et des dimensions territoriales, à différents niveaux d'organisation, d'espace et de temps (voir Fig. 1). 
L'irréductible incomplétude de la pensée humaine oblige à limiter les éléments de cet environnement qui seront pris en compte. Il serait hasardeux de préciser dans ce court chapitre comment raisonner ces choix. En revanche, nous indiquons ci-dessous comment réfléchir le périmètre de l'entité sociale, de/s activité/s, des registres de motivations et des ressources considérés. Ces choix dépendent toujours de la question posée, du contexte, de la connaissance disponible, et de choix disciplinaires et théoriques. Changer l'objectif de l'étude et la question conduit à modifier le périmètre du système d'activité considéré.

\section{Choix de l'entité sociale}

Le système d'activité s'applique à un acteur individuel ou collectif qui prend l'initiative des activités considérées et qui contribue à les mettre en ouvre, les gère et en tire un avantage. Mais il est aussi un prisme théorique par lequel le chercheur tente de se saisir d'une réalité complexe et de répondre à des questions. L'acteur de la combinaison d'activités peut être un individu, un couple, un ménage, un foyer, une famille, un petit collectif, une entreprise. Le choix de l'entité sociale considérée doit permettre d'analyser les relations qui s'établissent au sein de l'entité sociale (dans le cas d'un collectif), mais aussi d'examiner les relations de l'entité sociale avec ses ressources, ses activités, ses pratiques et son environnement. Une façon de raisonner ce choix est de repérer dans le contexte étudié quelles sont les unités de travail, de décision, de gestion, de résidence, de production, d'accumulation, d'épargne, de consommation et de prise de risque. Ces unités ne se superposent pas toujours et ne sont pas forcément les mêmes pour toutes les activités. Elles peuvent se transformer parfois rapidement dans le temps et dans l'espace. Tel est le cas de la haute vallée andine du Cañar (Équateur) où l'émigration clandestine engendre des familles transnationales dont les membres sont dispersés à l'échelle de la planète et séparés plusieurs années durant (notion d'" archipel d'activités " présentée dans [Vaillant, 2008]), sans pour autant faire disparaître un système d'activité familial multi-localisé dans lequel certaines décisions d'investissement agricole se prennent à New York et où les virements bancaires internationaux sont un outil de trésorerie.

Qui fait quoi ? Qui décide quoi ? Et comment les individus constitutifs de l'entité sociale définissent-ils leur place et leurs contributions aux activités, aux décisions et aux pratiques ? Comment qualifient-ils leurs rapports aux ressources et à l'environnement socio-écologique considérés ? Une recherche sur les transformations structurelles du rapport au travail des éleveurs français (Tab. 1) a par exemple conduit à étudier le système d'activité de l'individu, mais aussi celui du ménage (Fiorelli, 2010). Précisons que le choix d'un collectif comme entité sociale (ménage, famille, etc.) induit des contraintes méthodologiques de conduite des enquêtes qu'il importe de réfléchir soigneusement (avec qui conduire les entretiens ? comment ?). Ainsi, pour l'étude des relations entre les stratégies adaptatives et les pratiques techniques des producteurs de café et miel au Guatemala, les entretiens furent menés auprès des individus en charge de la prise de décisions techniques bien que les thématiques abordées relevaient souvent du niveau familial. Ces biais assumés doivent être explicités et pris en compte lors de l'interprétation des enquêtes (Bathfield, 2013). 


\section{Qualification des activités}

Définir le sens donné à l'activité revient à formuler un a priori sur ce que l'on observe mais parfois aussi sur la finalité de l'activité. À titre d'exemples, l'activité peut être un ensemble de tâches organisées au sein d'un processus (ergonomie, agronomie et autres sciences techniques), un processus qui concourt à la production ou à la transformation d'un produit ou d'un service (sociologie, économie), un processus identitaire, de socialisation ou cognitif, d'apprentissage ou de formation (sociologie, anthropologie, sciences de l'éducation). L'activité est un processus qui forme un tout que l'on peut isoler des autres processus. Elle peut toujours être réalisée de diverses façons (ce qui renvoie aux notions de techniques et de pratiques) et implique une prise de décision (dans le choix de l'activité mais aussi des pratiques) et des régulations (sociales, normatives, etc.). L'activité exprime une forme de régularité (opérationnelle, sociale, temporelle, spatiale, cognitive, etc.) qui n'est pas contradictoire avec son caractère fortement dynamique. Cela renvoie aux notions de transformation, de création, d'innovation et d'adaptation. Qualifier une activité humaine impose de définir ce qui en fonde le périmètre : le travail, un revenu, une production, la création de valeur, le sens donné, une compétence, un espace, une période, une qualification, une responsabilité, l'appartenance à une corporation, une socialisation, etc. Ces registres de qualification de l'activité ne se recouvrent pas toujours et peuvent se transformer dans le temps. Dans de nombreux contextes, ces activités sont aussi très dépendantes de statuts (sociaux, juridiques, fiscaux) qu’il convient de repérer. Il va de soi que les activités de productions de biens et services autoconsommés sont essentielles dans l'équilibre du revenu et méritent souvent d'être caractérisées et interprétées dans l'articulation entre activités " professionnelles " et activités " domestiques ", notamment pour comprendre les problématiques de subsistance et d'organisation du travail.

À titre d'exemple (Tab. 1), les recherches conduites au Guatemala sur les pratiques techniques dans les caféières de producteurs familiaux conduisent à analyser, de façon privilégiée, les activités de création de biens et de services susceptibles d'être échangés (sur un marché ou non), les études universitaires et les principales activités festives. En effet, trois fêtes annuelles rythment la vie sociale, commerciale et technique des producteurs. Selon les problématiques et les terrains, il peut aussi être judicieux de ne pas recourir à des qualificatifs d'activité ou de forme d'activité qui délimitent des corporations défendant les positions et les acquis de certains secteurs de l'économie. C'est ainsi qu'en France, l'activité « agricole » recouvre des activités de production, transformation, commercialisation, tourisme et de service sans pour autant relever d'un statut de pluriactivité. 


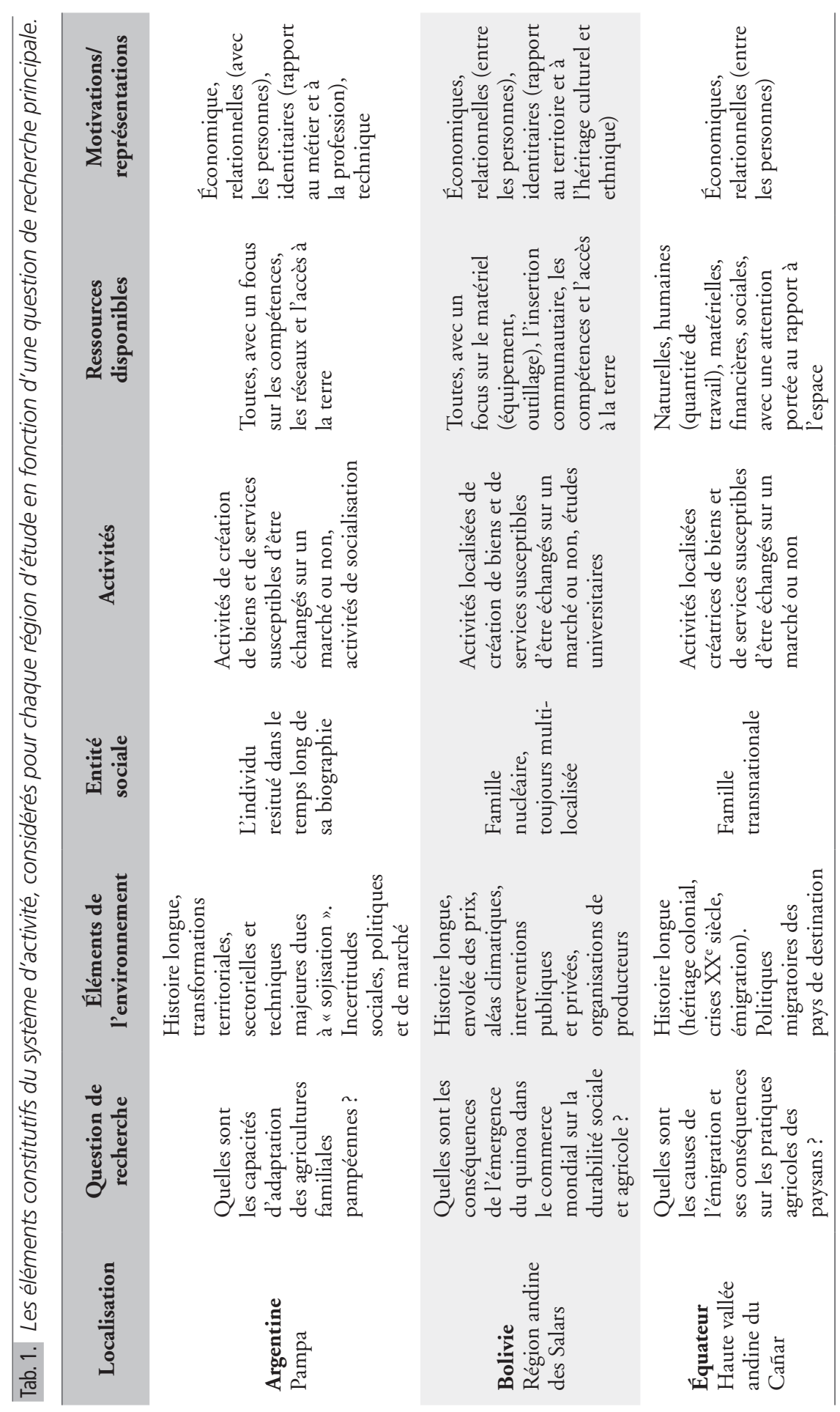




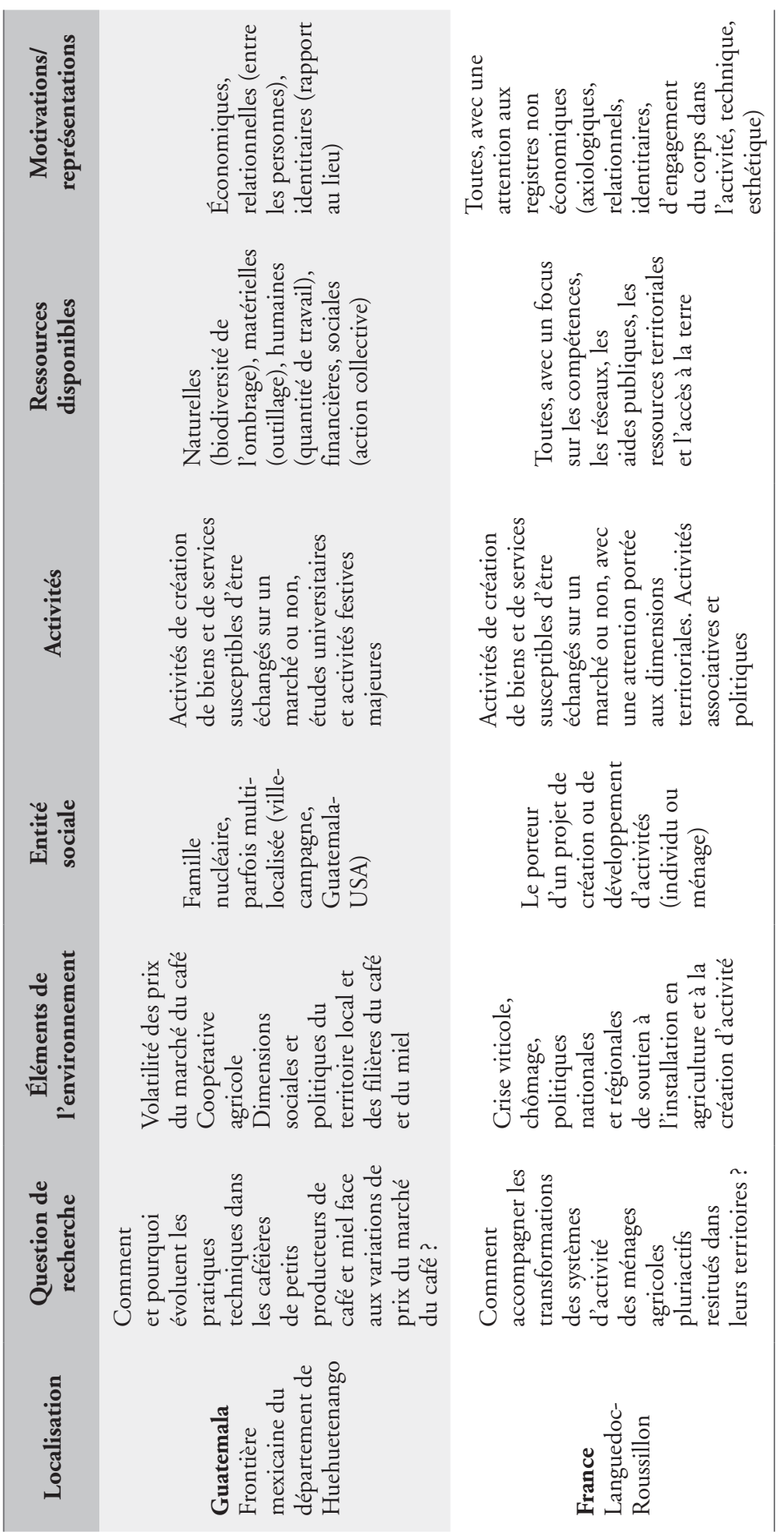




\section{Les registres de motivations et de représentations}

La mise en œuvre d'une combinaison d'activités par une entité sociale implique un équilibre ou une mise en tension entre plusieurs motivations, aussi qualifiées de « rationalités subjectives » par certains psychosociologues (Dejours, 1998). Fiorelli $e t$ al. (2010) confirment que « le travail ne sert pas qu’à produire et à gagner de l'argent, il permet de se sentir vivant, de se construire en tant qu'homme». Ces motivations président au choix des activités, des pratiques et au sens que l'entité sociale leur donne. Par ailleurs, nous considérons que l'acteur ne décide qu'en fonction de ses représentations et pas en fonction d'une information "pure et objective ». Ses représentations s'exercent à tous les niveaux : sur lui-même, sur ses ressources, ses activités, son environnement, sur ce qu'il estime des propriétés de son système (robustesse, vulnérabilité), etc. Le couple motivations/représentations est au cœur du processus décisionnel qui est rarement instantané lorsqu'on s'intéresse aux activités et aux pratiques. Nous reconnaissons les sept registres de motivation suivants (Fig. 1) :

- la motivation "économique " se réfere à l'intention d'optimiser l'usage de ressources limitées, au projet de création et d'échange de valeur sous forme de biens et de services (sur un marché ou non), à des logiques de production, de répartition, de consommation et d'accumulation ;

- la motivation "axiologique " renvoie aux valeurs morales et idéologiques de l'entité sociale, à sa vision du monde, à sa conception du bien et du mal, à une éthique de métier et/ou à une recherche de bien commun qui peuvent légitimer, justifier ou expliquer une activité, une pratique, une décision (Macombe, 2005) ;

- la motivation « relationnelle » qualifie les intentions d'interactions avec les autres hommes et avec les animaux, aux formes d'investissement affectif, au dessein de se produire, de vivre et de travailler ensemble (Fiorelli, 2010);

- la motivation identitaire renvoie à un registre de choix et de légitimation de l'activité et des pratiques du type "être ou ne pas être " par lequel l'entité sociale se reconnaît ou non. Cette motivation peut s'exprimer dans tous les champs de l'identité (professionnelle, territoriale, ethnique, religieuse, etc.). À titre d'exemple, dans son rapport au territoire, l'acteur exprime (ou non) un « je suis d'ici " (Sencébé, 2004). Les caractéristiques réelles et imaginées des territoires jouent un rôle important dans la mise en œuvre ou la transformation d'une situation d'activité ;

- la motivation « de l'engagement du corps " (Dejours, 1998) se réfere au « corps vécu ", au rapport physique et sensible à l'activité, à la pratique ou au travail. Elle traduit notamment des expressions de pénibilité, de stress ou au contraire de plaisir corporel et psychique ;

- la motivation " technique " renvoie au plaisir ou au déplaisir du geste et de la performance technique ;

- la motivation " esthétique » est assise sur le sentiment du beau (du paysage, du geste, du produit, etc.), et forme aussi un registre de choix et de légitimation des décisions prises concernant les activités et les pratiques. 
Bien entendu, l'individu n'exprime jamais ses motivations selon cette décomposition artificielle, ni sur tous ces registres. La liste est d'ailleurs probablement incomplète. Elle invite en tout cas à être attentif à la complexité des logiques d'action et des justifications de l'acteur pour comprendre ses décisions et ses pratiques. Elle implique de se doter de méthodologies permettant de les repérer. Les synergies et les tensions entre ces motivations permettent de comprendre les décisions, les arbitrages et les compromis, les choix d'activités et leur dimensionnement, les pratiques techniques et organisationnelles.

Certaines motivations ont plus d'importance que d'autres selon les problématiques posées, les contextes socio-économiques et culturels, et selon les marges de manœuvre auxquelles les acteurs peuvent prétendre. Pour délimiter les contours du système d'activité, il est impératif de caractériser et de justifier les motivations que le chercheur décidera d'examiner ou non. Ces choix sont trop souvent implicites (et réduits à la motivation "économique " du type Homo œeconomicus). Ils sont pourtant essentiels pour asseoir la rigueur d'un raisonnement scientifique et éviter le piège idéologique. De façon générale, le choix de motivations considérées dans l'étude dépend de l'échelle temporelle, sociale et spatiale d'analyse. La caractérisation d'une dynamique territoriale et d'une diversité des systèmes d'activité à l'échelle de la petite région, où s'exprime le poids des héritages socio-culturels et politiques et qui n'est pas la somme des choix individuels, limite l'intérêt d'un recours à un large spectre de motivations (Tab. 1 : voir études de cas en Équateur et en Bolivie). En revanche, l'analyse des intentions et des pratiques au niveau du projet que formule un individu nécessite d'examiner une large diversité de motivations (Tab. 1 : voir études de cas en France, en Argentine, en Bolivie et au Guatemala). Dans le cas du travail mené au Guatemala (Bathfield, 2013), les motivations considérées furent analysées au regard des stratégies des producteurs face à l'incertitude sur le temps long. Ainsi, sur la base d'une révision de littérature (Gasselin \& Bathfield, 2013) et d'une analyse des trajectoires de pratiques, nous nous sommes intéressés aux postures des producteurs sur le temps long face (i) à la dispersion du risque économique, (ii) à la diversification des activités, (iii) à la production pour l'autoconsommation, (iv) à l'action collective, (v) à l'ambition technique, (vi) à l'endettement, (vii) au changement de conduite, (viii) à l'épargne, (ix) à l'accroissement des moyens de production.

\section{Les ressources disponibles}

Nous considérons différents types de ressources disponibles, en se concentrant autant sur les questions de propriété que sur celles d'accès (Fig. 1) :

- "humaines " : qui renvoient aux dimensions qualitatives et quantitatives du travail, à la santé, à l'âge, au genre, aux savoirs et savoir-faire, etc. ;

- " naturelles » : terre, fertilité, eau, ressources génétiques, etc. ;

- matérielles : équipements, bâtiments, outillages, etc. ;

- financières : réserves d'argent facilement accessibles ; 
- informationnelles : les moyens de s'informer et l'information technique, économique, etc. mais aussi la connaissance des formes de coordination telles que les conventions, normes, règles ;

- identitaires : ethnicité, héritages culturels et professionnels, etc. ;

- ou sociales : notamment l'autorité (charismatique, légale, traditionnelle), l'insertion dans un réseau ou une organisation sociale (technique, commerciale ou autre), les formes de solidarité auxquelles l'acteur peut prétendre, etc.

D'autres classifications peuvent être pertinentes selon les problématiques posées, par exemple les ressources territoriales, institutionnelles, patrimoniales, etc. En Équateur (Vaillant, 2013), la ressource spatiale a été introduite pour bien rendre compte du fait que « l'espace n'est pas une simple étendue matérielle des supports des pratiques [...] mais une ressource sociale hybride et complexe mobilisée et ainsi transformée dans, par et pour l'action » (Lussaut, cité par Cortes, 2008). Si cette ressource est entendue comme l'ensemble des lieux aux potentialités spécifiques et de diverses natures qu'une entité sociale valorise via la mobilité, elle renvoie d'abord à la conception des pratiques qui tirent parti des potentialités agricoles de terrains disséminés à des altitudes différentes ; le degré de dispersion entre les écosystèmes étagés constituant ainsi une ressource (inégalement distribuée) que les paysans s'efforcent d'exploiter de façon à minimiser le risque agricole, étaler les périodes de travail au long de l'année et diversifier les productions.

Ces ressources sont variables dans le temps, en interaction, inégalement mobilisées selon l'activité considérée et rarement substituables. Elles peuvent dépendre principalement de l'entité sociale (ressources internes) ou former les conditions d'exercice des activités (ressources externes). Elle ne se capitalisent pas toujours (ainsi l'identité ethnique héritée ne fait pas l'objet d'échange ni d'accumulation). D'où notre choix de ne pas utiliser le terme de " capitaux ». Bien entendu, toutes les ressources n'ont pas la même importance dans les processus décisionnels, d'action et de création de valeur et de sens. Les ressources stratégiques changent selon les sociétés, les périodes historiques, les moments du parcours de vie, les activités, etc. Elles dépendent, en particulier, de la construction historique des relations sociales et des modalités de contrôle des ressources. La compréhension des pratiques et des décisions de l'acteur suppose d'avoir caractérisé ce qu'il reconnaît comme valeurs produites de l'activité, et donc ce qui fait ou non ressource pour lui.

\section{Fonctionnement et dynamique du système d'activité}

Par essence, le système d'activité oblige à prêter une attention toute particulière aux interactions qui le traversent (Fig. 1), qu'il s'agisse des interactions entre activités (produits, temps, espace, revenu, risque, sens, etc.), entre les ressources (concurrences, complémentarités, synergies, etc.), entre les divers éléments de la décision (motivations, représentations, stratégies, etc.) ou encore entre le système d'activité et son environnement politique et normatif, territorial et sectoriel. La crise écologique, financière et sociale qui ébranla l'Équateur à la fin du $\mathrm{XX}^{\mathrm{e}}$ siècle, les attentats 
du 11 septembre 2001 (à l'origine du très répressif USA Patriot Act) et la récente crise des subprimes constituent des évènements majeurs qui ont bouleversé l'environnement des familles paysannes de la haute vallée du Cañar et les ont contraint à modifier en conséquence leurs systèmes d'activité (Vaillant, 2013). Le système d'activité est pensé dans une dynamique continue qui implique d'interroger une coévolution du système et de son environnement : il s'agit non seulement de l'histoire de l'entité sociale et de ses activités mais aussi de celle de l'environnement, dans ses dimensions politiques, socio-économiques, techniques et écologiques. Cela suppose notamment de questionner les différentes échelles temporelles de l'action et de la prise de décision. Penser la dynamique du système d'activité amène aussi à en interroger les dimensions de vulnérabilité, de flexibilité (Bathfield et al., 2013 ; Gasselin et Bathfield, 2013), de résilience ou encore de durabilité (Terrier et al., 2013).

\section{Discussion-conclusion}

Le renouvellement du concept de système d'activité que nous proposons s'inscrit dans la continuité des fondements érigés par Paul et ses collaborateurs en 1994 : historicité, emboîtement d'échelles, attention portée autant à l'activité (pratiques, décisions) qu'aux ressources, jeu complexe des interactions, un regard non agricolo-centré. Il s'en distingue par un raffinement de l'analyse des activités (analyse du périmètre et du sens) et des ressources (sur une gamme plus large, internes/ externes), la reconnaissance d'une diversité de configurations de l'entité sociale (individu, ménage, famille, entreprise, etc.), l'affirmation d'une poly-rationalité de l'acteur, l'attention portée aux représentations de l'acteur, la reconnaissance d'une double temporalité à l'interface d'une micro-histoire singulière et d'une macro-histoire sociale, un environnement de l'activité et de l'entité sociale qui est à la fois un contexte et un produit de l'activité.

Malgré des sujets de recherche similaires, l'approche système d'activité se démarque du cadre conceptuel des Sustainable Rural Livelihoods (SRL) sur de nombreux aspects. L'approche SRL a été stabilisée en 1991 par le papier de référence de Chambers et Conway (1991). "Un livelihood comprend les capabilités, les actifs (y compris les ressources matérielles et sociales) et les activités comme moyen de subsistance. Un livelihood est durable quand il peut faire face à et se remettre de contraintes et de chocs, de maintenir ou d'améliorer ses capacités et ses biens, sans pour autant compromettre la base de ressources naturelles" (Scoones, 1998). L'objectif des SRL était de développer une perspective multidisciplinaire centrée sur les personnes afin d'établir des politiques et des actions de développement. Construit en parallèle avec une réflexion politique sur la notion de durabilité, le concept de SRL a été rapidement repris par de nombreuses institutions politiques, conduisant à une explosion de travaux sur les SRL. L'abondance d'études qui résultent de cet engouement a souvent conduit à une simplification du cadre SRL tout en soulignant les limites pratiques de ce cadre conceptuel (Scoones, 2009). En effet, certains auteurs (Gaillard 
et Sourisseau, 2009 ; Gasselin, 2012a ; O’Laughlin, 2004 ; Scoones, 2009) ont pointé la difficulté pour les chercheurs d'appliquer ce cadre ainsi que certaines de ses faiblesses telles que :

- le peu d'attention portée à l'entité sociale ${ }^{7}$ (qui n’est pas identifiée ni caractérisée dans les conceptualisations des SRL);

- la négligence d'une analyse de l'activité par l'examen des pratiques, qui conduit Scoones (2009 : p. 186) à dénoncer « une vision évolutionniste et normative du développement, où l'on distingue les « bonnes " et les « mauvaises " activités ";

- le manque de perspective systémique, qui amène à omettre l'analyse des propriétés émergentes et des interactions (entre activités, entre ressources, etc.) ;

- la considération de la substituabilité des différents capitaux (physique, financier, humain, social et naturel) qui conduit à négliger d'étudier le caractère plus ou moins stratégique des capitaux dans des jeux de pouvoirs et d'exclusion ;

- l'absence d'ancrage historique local et une lecture souvent apolitique des enjeux (la pauvreté devenant un problème de dotation et de combinaison de capitaux à l'échelle micro de la famille et de l'individu);

- une normalisation de la durabilité (présentée comme un outcome des SRL) par le biais d'indicateurs qui oublient le fait que la durabilité est un construit daté, social, cognitif et que ses enjeux sont toujours territorialisés ;

- la difficulté de prendre en compte "les ressources cachées » telles que les ressources culturelles ou même de reconnaître des ressources à gérer (telles que les ressources naturelles et identitaires) et non à capitaliser.

L'approche des systèmes d'activité constitue une autre manière de voir qui prétend éviter ces écueils, en mettant l'accent sur l'activité plutôt que sur les capitaux (la combinaison des activités fait système et définit sa cohérence). En outre, l'approche système d'activité n'est pas limitée aux personnes pauvres comme dans le cadre des SRL. Dans les systèmes d'activité, le cœur de l'analyse est la prise de décisions et les pratiques des acteurs tout en tenant compte de leur poly-rationalité dans une perspective systémique. Cette approche se démarque également en raison (i) de l'impératif de contextualisation du système et de son environnement, (ii) de la prise en compte de " ressources " et non de " capitaux ", (iii) de l'examen de l'historicité et (iv) des relations à l'environnement (à la fois contexte et produit de l'activité).

Le concept de système d'activité ainsi défini oriente les travaux de catégorisation des formes sociales et techniques observées dans les diverses expressions de la ruralité, et pas seulement de l'agriculture. Il conduit à réinterroger, de manière systémique, l'activité, les pratiques et les décisions, ainsi que les interactions liant activités, ressources pour l'action et la décision, représentations et motivations multiples de

7. L'approche par les SRL considère l'individu dans ses capitaux et ses capabilités (Sen, 2008), i.e. la possibilité effective qu'a un individu de mobiliser les capitaux disponibles et de prendre des décisions. Ce faisant, elle laisse dans l'ombre certaines dimensions qui déterminent aussi la décision et le rapport à l'activité, notamment le fait que l'acteur ne se réduit souvent pas à un individu mais est une entité sociale composée d'individus en interrelations. 
l'acteur. Il invite à comparer les processus de transformations observés et permet de revisiter les formes d'articulation d'une entité sociale avec les niveaux d'organisation supérieurs, qu'il s'agisse de marchés, de territoires, de politiques publiques ou de dispositifs de normes. Il implique d'en interroger l'historicité dans une lecture où l'entité sociale est à la fois (i) le produit d'une micro-histoire et d'une histoire sociétale, (ii) une entité qui s'adapte (ou non) à des régimes d'action, de perturbation et d'incertitude, mais aussi (iii) un acteur des processus socio-économiques, politiques et environnementaux, contributeur du fait technique et social, dans des organisations, dispositifs et réseaux.

Le système d'activité pose ainsi une triple temporalité (micro-histoire individuelle, macro-histoire sociale, processus adaptatifs) alors que le SRL est souvent interprété dans une perspective anhistorique et pose une durabilité statique (pas de prise en compte de l'adaptabilité) et normative (les paramètres de durabilité sont prédéterminés). Lapplication du concept de système d'activité aux questions de durabilité (Terrier et al., 2013) implique (1) une posture constructiviste et compréhensive pour qualifier les enjeux, critères et indicateurs de durabilité et (2) la distinction entre une durabilité restreinte (la durabilité du système pour lui-même) et une durabilité étendue (la contribution du système d'activité à la durabilité de niveaux d'organisation supérieurs).

Le concept de système d'activité est à l'interface de plusieurs disciplines : agronomie, zootechnie, économie, sociologie, sciences de gestion, ergonomie, géographie, anthropologie, histoire. Il nous semble pouvoir contribuer à renouveler le regard porté sur l'exploitation agricole. Il déplace et élargit les études portées par le concept de système de production agricole, et permet d'interroger les recompositions contemporaines de l'exploitation au Nord comme au Sud (délégation d'activité, contractualisation, recomposition de l'action collective de proximité, progressivité de l'installation, pluriactivité, dilution du caractère familial, entrepreneurialisation, reconfigurations des mobilités géographiques, nouveaux régimes de connaissances et d'innovation, etc.) et dans le renouvellement de ses enjeux au-delà des dimensions strictement agricoles (alimentaires, santé, agroécologiques, climatiques, relations à la ville, etc.). Soulignons également que le concept de système d'activité n'est pas qu'un instrument analytique visant à une meilleure intelligibilité des situations. Il peut aussi être mobilisé dans une démarche d'accompagnement, par exemple comme le support d'une interconnaissance des membres d'une coopérative d'activité et d'emploi en vue de susciter et d'organiser l'action collective. Pour autant, nous attirons l'attention du lecteur sur la tentation totalisante et l'exhaustivité fictive que suscite parfois l'approche systémique, comme d'autres auteurs l'ont déjà souligné (Olivier de Sardan, 1995 ; Lugan, 2000), et sur les risques associés (dérives mécanistes, négligence du sensible, contournement de l'épreuve philosophique, le social fonctionnalisé, etc.). 


\section{Bibliographie}

Albaladejo, C., Arnauld de Sartre, X., Gasselin, P., 2012. Agriculture entrepreneuriale et destruction du travail dans la Pampa argentine, Études Rurales, 190, 177-192.

Albaladejo, C., Gasselin, P., Goulet, F., 2011. Fragmentations agricoles et rurales dans la Pampa argentine : aux confins du territoire ? in Caron, P., Hervieu, B., Hubert, B. (Eds), Colloque "Agricultures et alimentations dans un monde globalisé ", Cerisy-la-Salle, 22-28 septembre 2011.

Arendt, H., 1994. La condition de l'homme moderne, Paris, Pocket.

Aubry, C., 2007. La gestion technique des exploitations agricoles : composante de la théorie agronomique, Mémoire d'Habilitation à Diriger les Recherches, Toulouse, Institut National Polytechnique de Toulouse.

Bathfield, B., 2013. Estrategias adaptativas y gestiones técnicas de los pequeños productores de café y miel en los altos de Guatemala, Thèse de doctorat en "Écologie et développement durable ", San Cristobal de Las Casas, Chiapas, Mexique, El Colegio de la Frontera Sur (ECOSUR).

Bathfield, B., Gasselin, P., López-Ridaura, S., Vandame, R., 2013. A flexibility framework to understand the adaptation of small coffee and honey producers facing market shocks, The Geographical Journal, 179, 4, 356-368.

Bebbington, A., 1999. Capitals and Capabilities : A Framework for Analyzing Peasant Viability, Rural Livelihoods and Poverty, World Development, 27, 12, 2021-2044.

Boltanski, L., Chiapello, E., 1999. Le nouvel esprit du capitalisme, Paris, Gallimard.

Boserup, E., 1970. Évolution agraire et pression démographique, Paris, Flammarion. (Édition originale en anglais : The Conditions of Agricultural Growth. The Economics of Agriculture under Population Pressure. Chicago, Adline publishing, 1965.)

Bourdieu, P., 1980. Le sens pratique, Paris, Éditions de Minuit.

Brossier, J., Vissac, B., Le Moigne, J.-L. (Eds), 1990. Modélisation systémique et système agraire. Décision et organisation, Paris, INRA.

Caron, P., Reig, E., Roep, D., Hediger, W., Cotty, T.L., Barthelemy, D., Hadynska, A., Hadynski, J., Oostindie, H.A., Sabourin, E., 2008. Multifunctionality : refocusing a spreading, loose and fashionable concept for looking at sustainability ? International Journal of Agricultural Resources, Governance and Ecology, 7, 4/5, 301-318.

Cellier, J.-M., Marquié, J.-C., 1980. Système d'activités et régulations dans l'exploitation agricole, Le Travail humain, 43, 2, 321-336.

Chambers, R., Conway, G., 1991. Sustainable rural livelihoods : practical concepts for the 21st century, IDS Discussion Paper, 296.

Chayanov, A.-V., 1990. L'organisation de l'économie paysanne, Paris, Librairie du Regard.

Chia, E., Dugué, P., Sakho-Jimbira, M.-S., 2006. Les exploitations agricoles familiales sont-elles des institutions? Cahiers Agricultures, 15, 6, 498-505. 
Cochet, H., 2011. L'agriculture comparée, Paris, éditions Quae.

Cochet, H., Devienne, S., Dufumier, M., 2007. L'agriculture comparée, une discipline de synthèse ? Économie Rurale, 297-298, 99-112.

Cortes, G., 2008. Migrations, espaces et développement. Une lecture des systèmes de mobilité et des constructions territoriales en Amérique latine, Rapport présenté pour l'obtention de l'habilitation à diriger des recherches, vol. 3 (synthèse des travaux et des positionnements de recherche), Université de Poitiers.

Crozier, M., Friedberg, E., 1977. L'acteur et le système, Paris, Éditions du Seuil.

Curie, J., Hajjar, V., Marquié, H., Roque, M., 1990. Propositions méthodologiques pour la description des systèmes d'activités, Le travail humain, 53, 2, 103-118.

Darré, J.-P., 1999. La production de connaissance pour l'action - Arguments contre le racisme de l'intelligence, Paris, MSH-INRA.

Dedieu, B., Laurent, C., Mundler, P., 1999. Organisation du travail dans les systèmes d'activités complexes, Économie Rurale, 253, 1, 28-35.

De Janvry, A., Sadoulet, E., Zhu, N., 2005. The Role of Non-Farm Incomes in Reducing Rural Poverty and Inequality in China, Berkeley, Department of Agricultural and Resource Economics, University of California.

Dejours, C., 1998. Travailler n'est pas déroger, Travailler, 1, 5-12.

Dufumier, M., 2006. Diversité des exploitations agricoles et pluriactivité des agriculteurs dans le Tiers Monde, Cahiers Agricultures, 15, 6, 584-588.

Ellis, F., 1998. Household strategies and rural livelihood diversification, Journal of Development Studies, 35, 1, 1-38.

Ellis, F., 2000. Rural livelihoods and diversity in developing countries, New York, Oxford University Press.

Farrington, J., Carney, D., Ashley, C., Turton, C., 1999. Sustainable livelihood in practice : early applications of concepts in rural areas, ODI Natural resources perspectives, 42.

Fiorelli, C., 2010. L'aménagement des conditions de vie au travail des éleveurs : proposition d'un cadre d'analyse des relations entre rapport subjectif et organisation du travail en élevage. Etude de cas chez des éleveurs pluriactifs, Thèse de doctorat délivré par l'Institut des sciences et industries du vivant et de l'environnement (AgroParisTech), spécialité : zootechnie des systèmes d'élevage, Paris, AgroParisTech-INRA.

Fiorelli, C., Dedieu, B., Porcher, J., 2010. Un cadre d'analyse des compromis adoptés par les éleveurs pour organiser leur travail, Cahiers Agricultures, 19, 5, 383-390.

Gaillard, C., Sourisseau, J.-M., 2009. Système de culture, système d'activité(s) et rural livelihood : enseignements issus d'une étude sur l'agriculture kanak (Nouvelle-Calédonie), Journal de la Société des Océanistes, 129, 2, 279-294.

Gasselin, P., 2011. Co-conception d'une politique publique pour une région arrière du développement : le cas de la pluriactivité en Languedoc-Roussillon, Pour, numéro spécial "Innovations et alternatives en agricultures », 212, 155163. 
Gasselin, P., 2012a. Amender les "Sustainable Rural Livelihoods » est-il pertinent ? Discussion de l'exposé de Pierre-Marie-Bosc et Jean-Michel Sourisseau intitulé " Décrire la diversité des systèmes d'activités des ménages ruraux ", in UMR ARTDev (Ed), Les jeudis d'Art-Dev-Les journées TerreDev : "Des ménages aux territoires - Concepts et méthodologies ", Montpellier, jeudi 12 juillet 2012.

Gasselin, P. (Ed), 2012b. Rapport scientifique final du projet "Insertion territoriale des systèmes d'activités des ménages agricoles" (Intersama) dans le cadre du programme "Pour et Sur le Développement Régional " (PSDR3) en LanguedocRoussillon, Montpellier, INRA.

Gasselin, P., Bathfield, B., 2013. La flexibilidad de los sistemas de actividad : un marco de análisis de las propiedades y de los procesos adaptativos de las actividades de los agricultores, in Gasselin, P., Cloquell, S., Mosciaro, M. (Eds), Adaptación y transformaciones de las agriculturas pampeanas al inicio del siglo XXI, Buenos Aires, Ciccus.

Gasselin, P., Puschiasis, O., Bourliaud, J., Métais, S., 2010. La fertilité revisitée : innovation et crise des agricultures de l'Altiplano bolivien, in Coudel, E., Devautour, H., Toussaint Soulard, C., Hubert, B. (Eds), International Symposium on "Innovation \& Sustainable Development in Agriculture and Food" - ISDA 2010, Montpellier - France, 28 June-1 July 2010.

Gasselin, P., Vaillant, M., 2010. La migración como elemento clave de los sistemas de actividades campesinos para enfrentar la incertidumbre. Análisis comparativo de dos regiones rurales andinas (Bolivia, Ecuador), in MRdT Bolivia (Ed), III Congreso Mundial de la Quinoa, Oruro, Bolivia - March 16-18, 2010.

Gasselin, P., Vaillant, M., Bathfield, B., 2012. The activity system. A position paper, in IFSA (Ed), 10th European IFSA Symposium "Producing and reproducing farming systems: New modes of organization for the sustainable food systems of tomorrow». Workshop 1.3 "Understanding agricultural structural changes and their impacts, to support inclusive policy dialogue and formulation", Aarhus University (Denmark), 1-4 July 2012.

Gumuchian, H., Pecqueur, B. (Eds), 2007. La ressource territoriale, Paris, Economica. Haggblade, S., Hazell, P., Reardon, T., 2007. Transforming the Rural Nonfarm Economy. Opportunities and Threats in the Developing World, Baltimore, The John Hopkins University Press.

Lanneau, G., Baubion-Broye, A., Bordes, O., Curie, J., Hajjar, V., 1987. Processus régulateurs des systèmes d'activités et changements socio-techniques, Bulletin de psychologie, XL, 379, 359-365.

Laurent, C., Cartier, S., Fabre, C., Mundler, P., Ponchelet, D., Rémy, J., 1998. L'activité agricole des ménages ruraux et la cohésion économique et sociale, Économie Rurale, 224, 12-21.

Lugan, J.-C., 2000. La systémique sociale, Paris, Presses Universitaires de France.

Macombe, C., 2005. Une méthode pour détecter les éthiques de métier, Management \& Avenir, 4, 6, 63-84. 
Malézieux, E., Moustier, P., 2005. La diversification dans les agricultures du Sud : à la croisée de logiques d'environnement et de marché, Cahiers Agricultures, 14, 4, 375-382.

Mazoyer, M., Roudard, L., 1997. Histoire des agricultures du monde : du néolithique à la crise contemporaine, Paris, éditions du Seuil.

Morin, E., 1990. Introduction à la pensée complexe, Paris, éditions du Seuil

Mundler, P., 2013. Systèmes d'activités des ménages agricoles en Rhône-Alpes, des liens aux territoires par les produits, les services et l'emploi, Montagnes méditerranéennes, 25, 47-56.

Norman, D.W., 2002. The farming systems approach : a historical perspective, in IFSA (Ed), $17^{\text {th }}$ Symposium of the International Farming Systems Association "Small farms in an ever-changing world: Meeting the challenges of sustainable livelihoods and food security in diverse rural communities", Lake Buena Vista, Florida, USA - November 17-20, 2002.

O’laughlin, B., 2004. Book reviews, Development and Change, 35, 2, 385-403.

Olivier de Sardan, J.-P., 1995. Anthropologie et développement, Essai en socio-anthropologie du changement social, Paris, APAD-Karthala.

Ostrom, E., Baechler, L., 2010. La gouvernance des biens communs. Pour une nouvelle approche des ressources naturelles, Bruxelles, De Boeck.

Osty, P.-L., 1978. L'exploitation agricole vue comme un système. Diffusion de l'innovation et contribution au développement, Bulletin technique d'information (ministère de l'Agriculture et du Développement rural), 326, 43-49.

Paul, J.-L., Bory, A., Bellande, A., Garganta, E., Fabri, A., 1994. Quel système de référence pour la prise en compte de la rationalité de l'agriculteur : du système de production agricole au système d'activité, Les cahiers de la recherche-développement, 39, 7-19.

Polanyi, K., 1983. La Grande Transformation, aux origines politiques et économiques de notre temps, Paris, Gallimard.

Scoones, I., 1998. Sustainable rural livelihoods : a framework for analysis, Brighton, Working Paper 72, Institute for Development Studies.

Scoones, I., 2009. Livelihoods perspectives and rural development, Journal of Peasant Studies, 36, 1, 171-196.

Sebillotte, M. (Ed), 1996. Recherches-système en agriculture et développement rural : conférences et débats - Symposium international, Montpellier, France : 21-25 novembre 1994, Montpellier, CIRAD.

Sen, A., 2008. Éthique et économie, Paris, Puf.

Sencébé, Y., 2004. Être ici, être d'ici. Forme d'appartenace dans le Diois (Drôme), Ethnologie française, 2004/2, XXXVII, 23-29.

Terrier, M., Gasselin, P., Le Blanc, J., 2013. Assessing the Sustainability of Activity Systems to Support Households' Farming Projects, in Marta-Costa, A.A., Soares da Silva, E. (Eds), Methods and Procedures for Building Sustainable Farming Systems. Application in the European Context, Dordrecht (The Netherlands), Springer, 47-61. 
Vaillant, M., 2008. Formas espaciales y laborales de la movilidad campesina de Hatun Cañar : de la microverticalidad agro-ecológica a los archipiélagos de actividades, in Godard, H., Sandoval, G. (Eds), Migración transnacional de los Andes a Europa y Estados Unidos, Lima, IFEA - PIEB - IRD, 103-134.

Vaillant, M., 2013. L'araire ou la barque. Migrations, mondialisation et transformations agraires en région de haute montagne (Andes australes de l'Équateur), Thèse de doctorat délivré par l'Institut des sciences du vivant et de l'environnement (AgroParistech), spécialité agriculture comparée, Paris, AgroParisTech. 